\title{
Identifying scoliosis in population-based cohorts: development and validation of a novel method based on total body DXA scans
}

Taylor $\mathrm{HJ}^{1}$, Harding $\mathrm{I}^{2}$, Hutchinson $\mathrm{J}^{2}$, Nelson $\mathrm{I}^{2}$, Blom A ${ }^{1}, \mathrm{JH}_{\text {Tobias }}{ }^{1}, \mathrm{EM} \mathrm{Clark}^{1}$

${ }^{1}$ University of Bristol, UK. ${ }^{2}$ North Bristol NHS Trust, UK.

\section{Introduction}

- Scoliosis is lateral curvature of the spine $\geq 10^{\circ}$, as measured on a standing spinal radiograph (see radiograph), with the most common type being Adolescent Idiopathic Scoliosis (AIS)

- There are no published studies that have investigated determinants of scoliosis using a prospective study design, making the establishment of cause and effect difficult - Several large population-based cohorts exist throughout the world with a range of clinical, biological and genetics data already collected

- Spinal imaging with traditional radiographs is not generally collected in these cohorts because of the ethical issues surrounding radiation exposure in otherwise healthy populations

- However, total body DXA scans have been routinely collected at repeated time points for the study of determinants of bone size and density.

\section{Aims}

- To develop and validate a novel method of identifying scoliosis on total body DXA scans

\section{Methods \\ DXA Scoliosis Method (DSM)}

- scoliosis was identified by triaging to distinguish true curves from positioning errors (see Figure 1 ) - once positioning errors were excluded, a modifiedFerguson method was used to measure angles (see Figure 2)

Figure 2: Position of lines and site of angle measurement
with the DSM. The normal spine line (NSL) is placed by
identifying the centre of the spine at first rib and drawin dentifying the centre of the spine

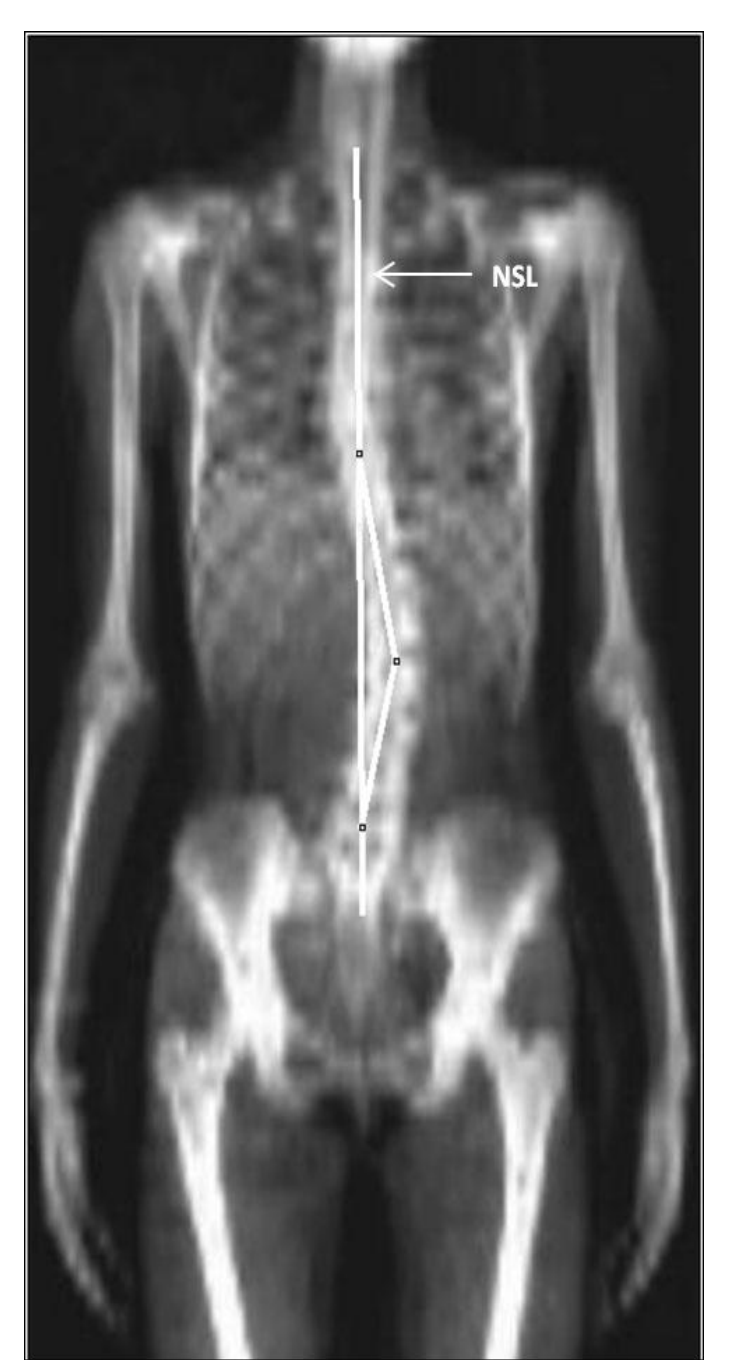

- additional data collected included site, direction and number of curves

Precision

- precision of the triage method was estimated by using data from 174 children from ALSPAC (see below) who underwent repeat DXA scans at aged 15, 2-6 weeks apart. The triage was applied to this set of 174 children with 2 scans and an unweighted Cohen's kappa statistic was calculated

- intraobserver variability of angle size measurement was assessed by calculating the coefficient of variation (CV) on 20 scans measured 5 times each

- interobserver variability of angle size measurement was assessed by the Bland-Altman method on 20 scans measured twice by two researchers ( $\mathrm{HT}$ and $\mathrm{EC}$ )

\section{Accuracy}

- angle size obtained by the DSM on DXA was compared to that obtained by Cobb method on spinal radiographs in 13 individuals with known scoliosis

Application of method to ALSPAC: Avon Longitudinal Study of Parents and Children

- ALSPAC is a large population-based birth cohort from southwest UK

- DXA scans had been previously obtained from 7298 participants at aged 9 and 5122 at aged 15

- the DSM was applied to these individuals to estimate scoliosis prevalence rates and curve patterns
Figure 1: Bristol DXA Scoliosis Method (DSM) triage

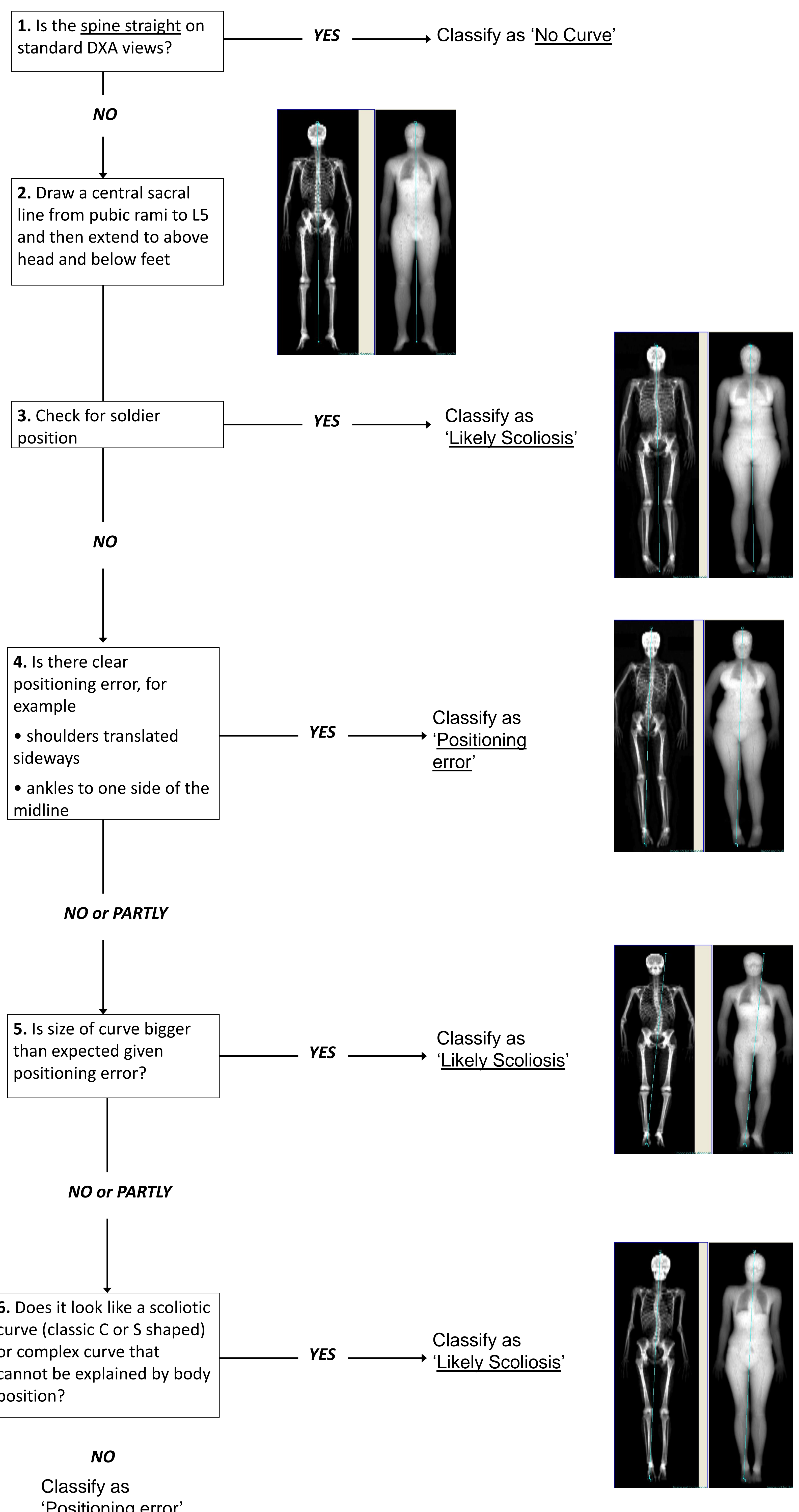

'Positioning error'

\section{Results: precision}

- there was substantial agreement for the triage method on repeat DXA scans taken 2-6 weeks apart: Kappa of 0.75 $(95 \% \mathrm{Cl} 0.55$ to 0.92$)$

- intraobserver variability (CV) for angle size was $10.9 \%$ (95\% Cl 8.4 to 13.5$)$

- of angle measurements carried out by 2 people (interobserver variability) $95 \%$ of were within $4.6^{\circ}$ of each other (see Figure 3)

Figure 3: Magnitude and distribution of differences in ment by 2 researchers. The $95 \%$ limits of agreement show the limits within which $95 \%$ of differences between measurements occur

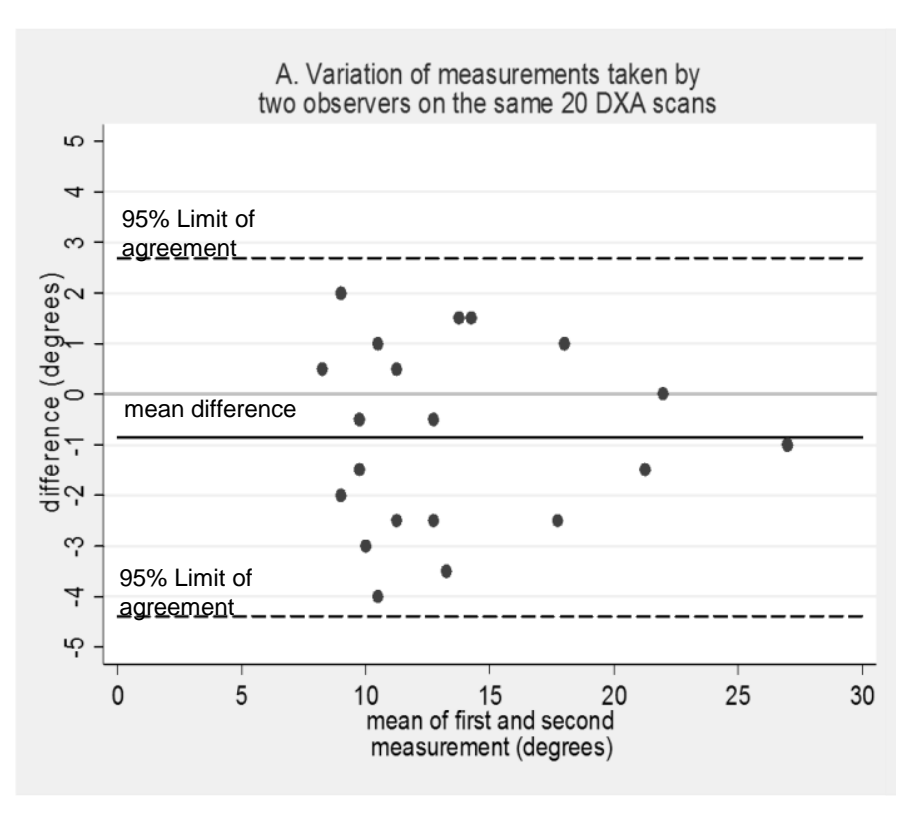

Results: accuracy

- mean angle size by our modified-Fergusson method on DXA was $28 \pm 15^{\circ}$ by the modified-Fergusson method on radiographs was $38 \pm 19^{\circ}$ and by the Cobb method on standing radiographs was $46 \pm 22^{\circ}$.

- mean reduction in angle size from supine DXA compared to standing radiographs was $11.7^{\circ}(30.8 \%)$

- mean reduction in angle size from using modifiedFergusson method compared to Cobb was $5.9^{\circ}(12.8 \%)$

Results: description of scoliosis in ALSPAC

using $\mathrm{a} \geq 10^{\circ}$ definition

- prevalence of scoliosis at aged 15 was 3.5\% (5.1\% in girls and $1.7 \%$ in boys). Of those with scoliosis at aged 15 , $76.8 \%$ were female

- mean + SD curve size at aged 15 was $15 \pm 7^{\circ}$

- of those with scoliosis at aged 15, $115(65 \%)$ had single curves, $54(30.5 \%)$ had double, and $8(4.5 \%)$ had more complex curves

- of those with scoliosis at aged 15, 41 (35.7\%) had thoracic and 74 (64.3\%) thoraco-lumbar curves

- of those with thoracic curves at aged $15,68.3 \%$ were right-facing

\section{Using $a \geq 6^{\circ}$ definition}

- as the DSM underestimates curve size by approximately $40 \%$ due to a combination of supine imaging plus use of a modified-Fergusson method we have also estimated prevalence using a $6^{\circ}$ cut-off

- prevalence of scoliosis at aged 15 was $5.9 \%$

- agreement using this lower cut-off was only moderate (kappa of $0.56,95 \% \mathrm{Cl} 0.41$ to 0.71 )

\section{Conclusion}

Radiograph showing scoliosis

identifying scoliosis from DXA scans

- comparison with prevalence data using more established techniques suggests our method provides valid estimates of scoliosis prevalence in population-based cohorts.
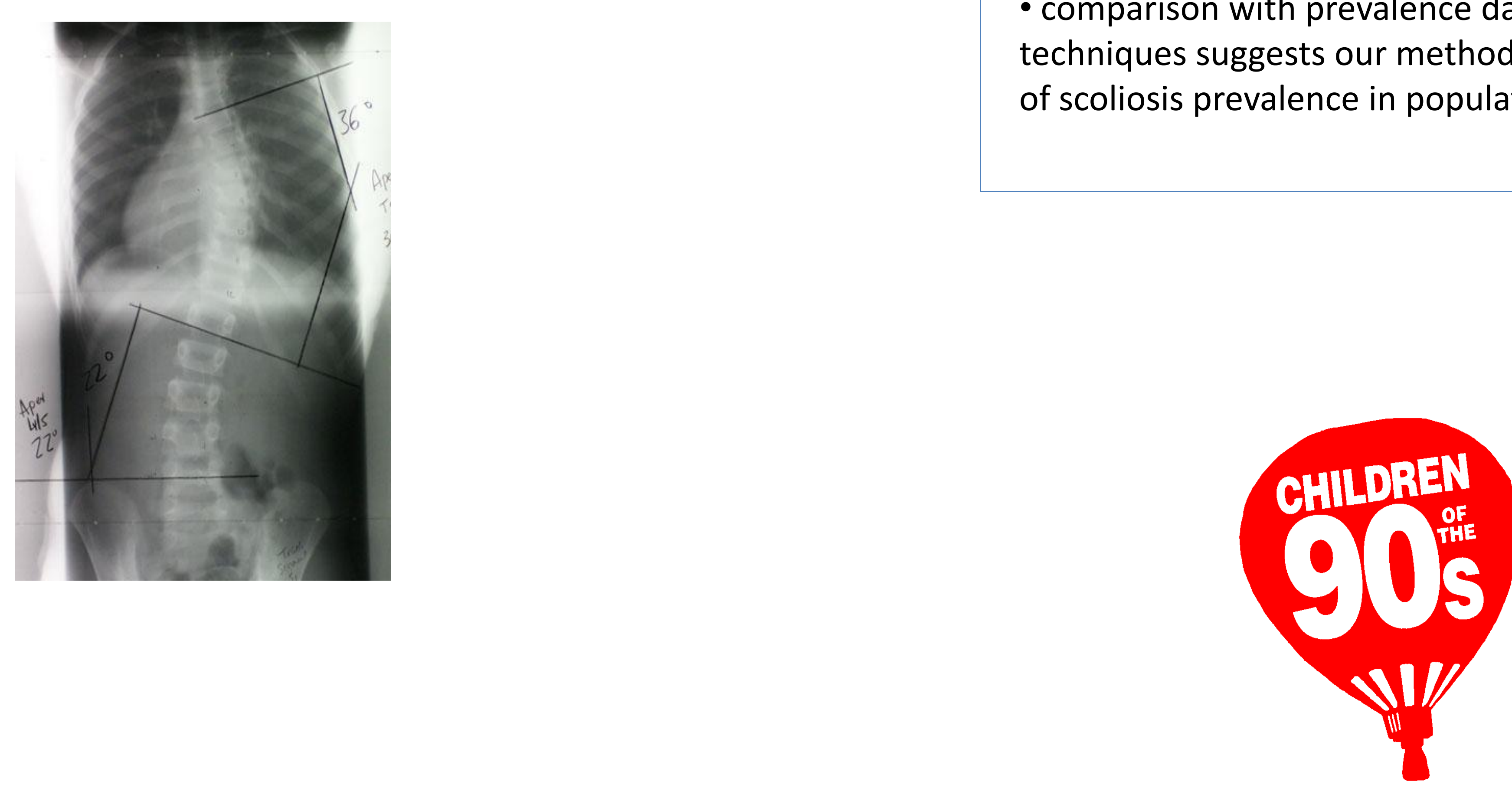